\title{
Efeito da Conexidade de Dados sobre o Valor Fenotípico Médio e a Variância Genética Aditiva 1
}

\author{
Antonio Policarpo Souza Carneiro2, 5 , Robledo de Almeida Torres ${ }^{3,6}$, Ricardo Frederico \\ Euclydes $^{3}$, Martinho de Almeida e Silva4, 6, Paulo Sávio Lopes 3,6 , Paulo Luiz Souza Carneiro ${ }^{2,6}$, \\ Rodolpho de Almeida Torres Filho', 6
}

\begin{abstract}
RESUMO - Dados simulados foram utilizados para verificar o efeito da conexidade sobre o valor fenotípico médio e a variância genética aditiva. O genoma simulado foi constituído de uma característica quantitativa governada por 500 locos. Simulou-se o efeito de rebanho ( 9 rebanhos) significativo a 5\% de probabilidade pelo teste F. Foram simulados arranjos de dados com 0, 15, 30, 60, 90 e 100\% de conexidade para herdabilidades 0,$10 ; 0,30$; e 0,60 e para diferentes tamanhos de progênie $(9,54$ e 90 progênies/reprodutor). A cada geração foram selecionados nove machos e o número de fêmeas selecionadas variou de acordo com o número de progênies considerado em cada arranjo de dados. A seleção foi efetuada em dez gerações, sendo este processo repetido por 30 vezes. O uso de dados com baixa conexidade reduziu o valor fenotípico médio, sendo que o maior efeito da conexidade ocorreu para baixa herdabilidade e tamanho reduzido de progênie. $\mathrm{O}$ efeito da conexidade sobre a variância genética aditiva foi pequeno.
\end{abstract}

Palavras-chave: avaliação genética, BLUP, seleção e simulação

\section{Data Connectedness Effect on Average Phenotypic Value and Additive Genetic Variance}

\begin{abstract}
Simulated data were used to verify the effect of the connectedness on the average phenotypic value and the additive genetic variance. The simulated genome was formed from a quantitative trait governed by 500 loci. Significance herd effect ( 9 herds) by $5 \%$ probability F test was simulated. Data sets with $0,15,30,60,90$ and $100 \%$ of connectedness for heritabilities $0.10,0.30$ and 0.60 and for different progeny sizes $(9,54$ and 90 progenies/sire) were simulated. In each generation nine males were selected and the number of selected females shifted according to the number of progenies considering in each data arrange. The selection was accomplished in ten generations, where the process was repeated 30 times. Data with smaller connectedness reduced the average phenotypic value, and the largest effect of the connectedness was for small heritability and small progeny size. The connectedness effect on the additive genetic variance was small.
\end{abstract}

Key Words: BLUP, genetic evaluation, selection and simulation

\section{Introdução}

Atualmente, com a expansão dos mercados e a maior competitividade estabelecidas numa economia globalizada, existe uma tendência mundial de as avaliações genéticas de animais serem realizadas em nível de país ou até mesmo entre países. Entre os fatores que afetam a acurácia das avaliações nacionais e internacionais, a conexidade dos dados tem sido citada por vários autores como um dos principais.

O efeito básico da seleção, em programas de melhoramento genético de qualquer espécie, é a mudança relativa das frequiências gênicas da população. Este efeito pode ser evidenciado pelas alterações observáveis, ao longo das gerações sob seleção, nos parâmetros populacionais, tais como a média dos valores fenotípicos e a variância genética.

A avaliação genética com alta acurácia é fator fundamental para a seleção eficiente dos melhores animais. Assim, o efeito direto da conexidade dos dados ocorrerá sobre a eficiência do processo seletivo, podendo este efeito ser quantificado pelas alterações na média dos valores fenotípicos e na variância genética das populações sob seleção, ao longo das gerações.

O conceito de conexidade depende do modelo considerado, fixo ou misto. Segundo SEARLE (1971), para modelos fixos de classificação cruzada, a conexidade entre dados existe quando todas as fun-

\footnotetext{
${ }^{1}$ Parte da tese de Mestrado do primeiro autor. Financiamento: CAPES/CNPq/UFV.

2 Estudante de Pós-graduação da UFV. 36571-000 - Viçosa-MG. E.mail: policarpoc@bol.com.br

3 Prof. da UFV. Depto. de Zootecnia - CCA, Viçosa-MG. E.mail: rtorres@mail.ufv.br; rbaja@mail.ufv.br; pslopes@mail.ufv.br

${ }^{4}$ Professor da UFMG, Belo Horizonte-MG. E.mail: martinho@vet.ufmg.br

${ }^{5}$ Bolsista da CAPES. E.mail: ds31954@correio.cpd.ufv.br

6 Bolsista do CNPq. E.mail: ds28686@correio.cpd.ufv.br
} 
ções lineares entre dois níveis quaisquer dos fatores considerados são estimáveis. Nos modelos mistos, a conexidade entre os dados é definida em termos da correlação entre os efeitos aleatórios relativos às observações efetuadas em diferentes níveis de efeitos fixos. Existindo esta correlação, novamente as comparações são passíveis de predição não-viesada, mas a variância do erro de predição (PEV) destas comparações é afetada pelo grau de conexidade dos dados (MARTINS, 1994).

No modelo misto, a média da PEV das comparações entre efeitos aleatórios pertencentes a diferentes níveis de efeitos fixos é uma medida lógica do grau de conexidade dos dados (KENNEDY e TRUS, 1993 e MARTINS, 1994). Entretanto, a obtenção dessas variâncias para grandes volumes de dados, como ocorre no melhoramento animal, é impossível, pois exige a inversão da matriz dos coeficientes das equações de modelos mistos.

FOULLEY et al. (1992), KENNEDY e TRUS (1993), MATHUR et al. (1999), entre outros, propuseram métodos alternativos para medir a conexidade entre dados usando medidas correlacionadas com a média da PEV das comparações.

Ainda não está definida claramente uma medida adequada do grau de conexidade dos dados e seus efeitos sobre as avaliações genéticas. Nas avaliações atuais, pouca importância tem sido dada à conexidade dos dados, e a ação dos melhoristas limita-se à aplicação de restrições que visam eliminar os dados totalmente desconectados.

O processo de simulação tem sido freqüentemente usado em estudos de problemas de conexidade de dados, sobretudo pela sua facilidade em gerar vários tipos de estruturas de dados com diferentes graus de conexidade (TONG et al., 1980; KENNEDY, 1981; ANALLA et al., 1995 e SIGURDSSON e BANOS, 1995).

O objetivo deste trabalho foi avaliar o efeito da conexidade de dados sobre o valor fenotípico médio e a variância genética aditiva, para conjunto de dados com diferentes números de progênie por reprodutor e para características com diferentes herdabilidades.

\section{Material e Métodos}

Os dados utilizados neste trabalho foram simulados pelo programa "Genesys" (Genetic System) desenvolvido por EUCLYDES (1996), escrito para o compilador FORTRAN.

O genoma simulado foi constituído de 15 pares de cromossomos. Quinhentos locos, distribuídos ao aca- so nos cromossomos, controlavam a característica quantitativa usada nesse estudo. Os locos quantitativos foram dialélicos, não possuíam desvios de dominância e nem interações entre si. Foi simulado o efeito aditivo de cada gene, tendo estes efeitos uma distribuição normal.

Foram simuladas três populações-base, cada uma constituídas por 1800 animais (900 machos e 900 fêmeas) com herdabilidades baixa, média e alta $(0,10 ; 0,30 ;$ e 0,60 , respectivamente) e, além do efeito fixo de sexo, foi simulado o efeito de rebanho com nove níveis. Os efeitos de rebanho foram simulados, de modo a produzir significância a $5 \%$ de probabilidade pelo teste F.

A partir de cada população-base, foram escolhidos aleatoriamente nove machos e determinado número de fêmeas para serem os pais das três populações iniciais, sendo simuladas populações com diferentes números de progênies/reprodutor: 9, 54 e 90 (Tabela 1).

A partir das populações iniciais, teve início o processo de avaliação genética dos animais, sendo selecionados nove machos e um número determinado de fêmeas, de acordo com o número de progênies/ reprodutor (Tabela 1). Este processo se estendeu ao longo de dez gerações e foram feitas 30 repetições para reduzir o efeito da flutuação gênica.

Os arranjos de dados foram simulados variando o número de rebanhos em que cada reprodutor tinha progênies. Foram simulados arranjos nos quais os reprodutores tinham progênies em 1, 2, 3, 6, 8 ou 9 rebanhos, obtendo assim, arranjos com $0,15,30,60$, 90 e $100 \%$ de conexidade média, respectivamente. O grau médio de conexidade para cada arranjo foi calculado como a proporção média de progênies dentro de cada rebanho que são descendentes de reprodutores comuns (MATHUR et al., 1999).

Tabela 1 - Número de animais selecionados em cada geração com base no número de progênies/ reprodutor (NP/R)

Table 1 - Number of animals selected in each generation according to number of progenies/sire (NP/S)

\begin{tabular}{lccc}
\hline NP/R & $\begin{array}{c}\text { Descendentes/Fêmeas } \\
\text { Descendents/Females }\end{array}$ & $\begin{array}{c}\text { Reprodutores } \\
\text { Sires }\end{array}$ & $\begin{array}{c}\text { Fêmeas } \\
\text { Females }\end{array}$ \\
\hline 9 & 3 & 9 & 27 \\
54 & 3 & 9 & 162 \\
90 & 3 & 9 & 270 \\
\hline
\end{tabular}




\section{Rev. bras. zootec.}

Nas Tabelas 2 e 3 são apresentados exemplos dos tipos de arranjos simulados com diferentes graus de conexidade: um arranjo com $0 \%$ de conexidade, reprodutores com progênies em um único rebanho, e outro com $30 \%$ de conexidade, em que os reprodutores tinham progênies em três rebanhos.

A seleção dos animais foi feita com base nas informações do próprio indivíduo, dos ascendentes (pai e mãe) e colaterais (irmãos completos e meioirmãos), uma vez que a matriz de parentesco continha apenas as informações de pedigree relativas às duas últimas gerações. Utilizou-se o modelo animal, descrito a seguir:

$$
\mathrm{y}_{\mathrm{ikk}}=\mu+\mathrm{r}_{\mathrm{i}}+\mathrm{s}_{\mathrm{j}}+\mathrm{a}_{\mathrm{ijk}}+\mathrm{e}_{\mathrm{ijk}}
$$

em que $y_{i k k}$ é observação do animal $i$, do rebanho j e do sexo $\mathrm{k} ; \mu$, constante associada a cada observação; $\mathrm{r}_{\mathrm{i}}$, efeito fixo de rebanho, com $\mathrm{i}=1,2,3, \ldots, 9$; $\mathrm{s}_{\mathrm{j}}$, efeito fixo de sexo, com $\mathrm{j}=1$ e $2 ; \mathrm{a}_{\mathrm{ijk}}=$ efeito aleatório do animal k, no rebanho i e do sexo j; $\mathrm{e}_{\mathrm{ijk}}$, erro aleatório associado a cada observação.

Em cada geração, foram calculados o valor fenotípico médio da população e a variância genética aditiva, com base nos valores genéticos dos indivíduos:

$$
\overline{\mathrm{P}}=\frac{\sum_{\mathrm{i}=1}^{\mathrm{n}} \mathrm{P}_{\mathrm{i}}}{\mathrm{n}}
$$

em que $\mathrm{P}_{\mathrm{i}}$ é o valor fenotípico do indivíduo i obtido pela soma do valor genético mais o efeito do ambiente sobre este indivíduo e n, o número total de indivíduos na população.

$$
\hat{\sigma}_{a}^{2}=\frac{\sum_{i=1}^{n} a_{i}^{2}-\left[\left(\sum_{i=1}^{n} a_{i}\right)^{2} / n\right]}{n-1}
$$

em que $\hat{\sigma}_{a}^{2}$ é o estimador da variância genética aditiva; $\mathrm{a}_{\mathrm{i}}$, o valor genético do indivíduo $i$; e n, o número total de indivíduos na população.

\section{Resultados e Discussão}

As médias dos valores fenotípicos observados na décima geração, em geral, aumentaram com o acréscimo do número de progênies e da herdabilidade da característica (Tabela 4).

Os menores valores fenotípicos médios foram observados para o arranjo de dados com $0 \%$ de conexidade para a maioria das herdabilidades e números de progênies considerados, exceto para a herdabilidade $0,60 \mathrm{e}$ considerando 54 e 90 progênies/reprodutor, em que não foi observado efeito da conexidade sobre o valor fenotípico médio das populações (Tabela 4).

Considerando a herdabilidade 0,10 , a redução no número de progênies causou maior diferença entre os resultados obtidos a partir de arranjos simulados com diferentes graus de conexidade. Para os arranjos com nove progênies, os maiores valores fenotípicos médios foram obtidos com a utilização dos dados com maior conexidade. No entanto, pequenas diferenças foram observadas nos valores fenotípicos a partir de $60 \%$ de conexidade. Também, para os arranjos com 15

\begin{tabular}{|c|c|c|c|c|c|c|c|c|c|}
\hline \multirow[b]{2}{*}{$\begin{array}{l}\text { Reprodutores } \\
\text { Sires }\end{array}$} & \multicolumn{8}{|c|}{$\begin{array}{c}\text { Rebanhos } \\
\text { Herds }\end{array}$} & \multirow[b]{2}{*}{9} \\
\hline & 1 & 2 & 3 & 4 & 5 & 6 & 7 & 8 & \\
\hline 1 & 90 & - & - & - & - & - & - & - & - \\
\hline 2 & - & 90 & - & - & - & - & - & - & - \\
\hline 3 & - & - & 90 & - & - & - & - & - & - \\
\hline 4 & - & - & - & 90 & - & - & - & - & - \\
\hline 5 & - & - & - & - & 90 & - & - & - & - \\
\hline 6 & - & - & - & - & - & 90 & - & - & - \\
\hline 7 & - & - & - & - & - & - & 90 & - & - \\
\hline 8 & - & - & - & - & - & - & - & 90 & - \\
\hline 9 & - & - & - & - & - & - & - & - & 90 \\
\hline
\end{tabular}

Tabela 2 - Arranjo com $0 \%$ de conexidade e 90 progênies/reprodutor Table 2 - Arrange with $0 \%$ of connectedness and 90 progenies/sire 
Tabela 3 - Arranjo com $30 \%$ de conexidade e 90 progênies/reprodutor

Table 3 - Arrange with $30 \%$ of connectedness and 90 progenies $/$ sire

\begin{tabular}{|c|c|c|c|c|c|c|c|c|c|}
\hline \multirow{3}{*}{$\begin{array}{l}\text { Reprodutores } \\
\text { Sires }\end{array}$} & \multicolumn{8}{|c|}{$\begin{array}{c}\text { Rebanhos } \\
\text { Herds }\end{array}$} & \multirow{3}{*}{9} \\
\hline & \multirow[t]{2}{*}{1} & \multirow[t]{2}{*}{2} & \multirow[t]{2}{*}{3} & \multirow[t]{2}{*}{4} & \multirow[t]{2}{*}{5} & \multirow[t]{2}{*}{6} & \multirow[t]{2}{*}{7} & \multirow[t]{2}{*}{8} & \\
\hline & & & & & & & & & \\
\hline 1 & 30 & 30 & 30 & - & - & - & - & - & - \\
\hline 2 & - & 30 & 30 & 30 & - & - & - & - & - \\
\hline 3 & - & - & 30 & 30 & 30 & - & - & - & - \\
\hline 4 & - & - & - & 30 & 30 & 30 & - & - & - \\
\hline 5 & - & - & - & - & 30 & 30 & 30 & - & - \\
\hline 6 & - & - & - & - & - & 30 & 30 & 30 & - \\
\hline 7 & - & - & - & - & - & - & 30 & 30 & 30 \\
\hline 8 & 30 & - & - & - & - & - & - & 30 & 30 \\
\hline 9 & 30 & 30 & - & - & - & - & - & - & 30 \\
\hline
\end{tabular}

Tabela 4 - Acurácia média do teste de progênie nas dez gerações, considerando arranjos de dados com 0, 15, 30, 60, 90 e $100 \%$ de conexidade

Table 4 - Average accuracy of the progeny test on the ten generations, for data arranges with 0,15, 30, 60, 90 and $100 \%$ of connectedness

\begin{tabular}{|c|c|c|c|c|c|c|}
\hline & \multicolumn{6}{|c|}{$\begin{array}{c}\text { Graus de conexidade } \\
\text { Degrees of connectedness }\end{array}$} \\
\hline & $0 \%$ & $15 \%$ & $30 \%$ & $60 \%$ & $90 \%$ & $100 \%$ \\
\hline Herdabilidade & \multicolumn{6}{|c|}{9 progênies } \\
\hline Heritability & \multicolumn{6}{|c|}{9 progenies } \\
\hline 0,10 & 14,30 & 17,49 & 23,12 & 27,78 & 27,01 & 28,31 \\
\hline 0,30 & 31,18 & 33,72 & 40,82 & 45,83 & 47,71 & 46,02 \\
\hline 0,60 & 39,46 & 46,57 & 49,22 & 55,97 & 53,98 & 58,09 \\
\hline Herdabilidade & \multicolumn{6}{|c|}{54 progênies } \\
\hline Heritability & \multicolumn{6}{|c|}{54 progenies } \\
\hline 0,10 & 47,21 & 57,77 & 58,32 & 66,32 & 65,41 & 67,07 \\
\hline 0,30 & 57,53 & 71,34 & 75,28 & 78,92 & 79,73 & 79,91 \\
\hline 0,60 & 65,62 & 76,04 & 78,63 & 80,93 & 83,28 & 83,69 \\
\hline Herdabilidade & \multicolumn{6}{|c|}{90 progênies } \\
\hline Heritability & \multicolumn{6}{|c|}{90 progenies } \\
\hline 0,10 & 46,09 & 63,49 & 65,94 & 67,34 & 67,46 & 68,51 \\
\hline 0,30 & 61,17 & 78,01 & 77,51 & 80,88 & 80,4 & 82,29 \\
\hline 0,60 & 73,36 & 81,45 & 84,85 & 84,37 & 84,71 & 84,57 \\
\hline
\end{tabular}

e $30 \%$ de conexidade, os resultados foram semelhantes. Para tamanhos de progênies menores que 9, espera-se que ocorram maiores diferenças entre os arranjos com 60,90 e $100 \%$ de conexidade.

A magnitude do efeito da conexidade dos dados sobre os valores fenotípicos observados foi influenciado pelo número de progênies e pela herdabilidade da característica. Os maiores efeitos da conexidade foram observados para o conjunto de dados com baixa herdabilidade e menor número de progênies (Tabela 4).

A metodologia dos modelos mistos sempre obtém o BLUP dos valores genéticos de todos animais, levando em consideração o cumprimento de algumas pressuposições, como a conexidade dos dados. Quando atendidas todas as pressuposições, o ganho genético é maximizado, uma vez que os melhores animais são identificados com maior acurácia. Para dados totalmente desconectados, arranjos com $0 \%$ de conexidade, os valores genéticos dos animais podem ser subestimados ou superestimados, provocando, assim, a seleção de animais que não seriam escolhidos, se os dados fossem bem conectados, levando à redução nos ganhos genéticos.

As diferenças observadas quando se comparam 
os arranjos com mesmo número de progênies, entre características de alta e baixa herdabilidades, podem ser explicadas pelo fato de o BLUP dar maior importância relativa à informação do próprio indivíduo que às informações dos seus parentes, quando se tratam de características de alta herdabilidade. Portanto, a distribuição das progênies em maior número de rebanhos e, conseqüentemente, o grau de conexidade dos dados não tiveram efeito sobre os valores fenotípicos médios para características de alta herdabilidade.

Quanto maior o número de descendentes por reprodutor, maior é a probabilidade de aparecer genótipos diferentes, provenientes das recombinações por crossing over e, consequientemente, aumenta a probabilidade do surgimento de indivíduos com valores genéticos superiores. Isto explica os maiores valores fenotípicos médios, observados para os arranjos de dados com maior número de progênies (Tabela 4). Também, a pressão de seleção, principalmente sobre os machos, foi maior para os arranjos com progênies mais numerosas. Uma vez que, independentemente do número de progênies, sempre foram selecionados nove machos a cada geração.

A redução percentual na variância genética aditiva na décima geração, em relação à primeira geração, variou aproximadamente de 15 a $43 \%$, de acordo com a herdabilidade da característica, o número de progênies/reprodutor e grau de conexidade dos dados. As maiores reduções foram observadas para característica de alta herdabilidade, progênies numerosas e arranjos com maiores graus de conexidade, revelando que neste casos o processo de seleção foi mais eficiente (Tabela 5 ).

EUCLYDES (1996) e CARNEIRO (1998), trabalhando com dados simulados pelo "Genesys", também observaram queda na variância genética aditiva ao longo das gerações. Esta redução provavelmente ocorreu, devido à fixação de alelos favoráveis, como resultado do processo de seleção, que visou aumentar o valor fenotípico médio da população (Robertson, 1986 e Falconer, 1981 citados por CARNEIRO, 1998).

A queda da variância genética aditiva, utilizando dados simulados pelo "Genesys", pode não corresponder à observada em situações reais. Uma das explicações para este fato é que o processo de simulação usado não leva em consideração as mutações e a introdução de novos genótipos que aumentam a variabilidade genética das populações. Portanto, os dados simulados apresentaram queda acentuada da variância genética aditiva, o que não seria esperado em populações reais submetidas a processos seletivos.

A menor redução na variância genética aditiva foi observada para o arranjo de dados com $0 \%$ de conexidade para todas as situações simuladas (Tabela 5). Isto é, provavelmente, conseqüência do menor ganho genético observado para este arranjo, quando comparado aos demais. Quanto menor o ganho genético, menor é a fixação de alelos e, portanto, menor é a queda na variância genética aditiva (EUCLYDES,1996).

Tabela 5 - Acurácia média do teste de performance nas dez gerações, considerando arranjos de dados com 0, 15, 30, 60, 90 e $100 \%$ de conexidade

Table 5 - Average accuracy of the performance test in the ten generations, for data arranges with 0,15, 30,60,90 and $100 \%$ of connectedness

\begin{tabular}{|c|c|c|c|c|c|c|}
\hline & \multicolumn{6}{|c|}{$\begin{array}{c}\text { Graus de conexidade } \\
\text { Degrees of connectedness }\end{array}$} \\
\hline & $0 \%$ & $15 \%$ & $30 \%$ & $60 \%$ & $90 \%$ & $100 \%$ \\
\hline Herdabilidade & \multicolumn{6}{|c|}{9 progênies } \\
\hline Heritability & \multicolumn{6}{|c|}{9 progenies } \\
\hline 0,10 & 15,79 & 17,47 & 18,27 & 21,19 & 22,36 & 21,71 \\
\hline 0,30 & 21,94 & 21,78 & 23,34 & 26,5 & 27,8 & 28,15 \\
\hline 0,60 & 30,87 & 31,65 & 33,13 & 35,27 & 37,4 & 36,87 \\
\hline Herdabilidade & \multicolumn{6}{|c|}{54 progênies } \\
\hline Heritability & \multicolumn{6}{|c|}{54 progenies } \\
\hline 0,10 & 16,82 & 21,26 & 19,80 & 22,36 & 22,36 & 23,05 \\
\hline 0,30 & 31,08 & 32,67 & 32,66 & 33,71 & 33,34 & 32,74 \\
\hline 0,60 & 46,07 & 45,12 & 44,10 & 44,99 & 45,55 & 44,77 \\
\hline Herdabilidade & \multicolumn{6}{|c|}{90 progênies } \\
\hline Heritability & \multicolumn{6}{|c|}{90 progenies } \\
\hline 0,10 & 7,93 & 15,35 & 15,27 & 15,62 & 16,34 & 17,05 \\
\hline 0,30 & 27,39 & 31,60 & 32,25 & 31,91 & 32,68 & 33,15 \\
\hline 0,60 & 49,97 & 50,01 & 50,25 & 50,29 & 50,72 & 51,32 \\
\hline
\end{tabular}




\section{Conclusões}

A baixa conexidade dos dados, principalmente o uso de dados totalmente desconectados, reduziu o valor fenotípico médio e, conseqüentemente, os ganhos genéticos obtidos. No entanto, a partir de determinado grau de conexidade, dependendo da herdabilidade da característica e do número de progênies, as respostas da seleção sobre o valor fenotípico médio tornaram-se semelhantes, com aumentos no grau de conexidade.

A herdabilidade da característica avaliada e o número de progênies tiveram maior influência no valor fenotípico médio que a conexidade dos dados. A importância da conexidade foi maior nas avaliações genéticas de animais com número reduzido de informações de parentes (descendentes ou colaterais) e para características de baixa herdabilidade. Nestes casos, devem-se tomar medidas que aumentem a conexidade dos dados, tais como alevar a troca de material genético entre os rebanhos, ou analisar separadamente os conjuntos de dados desconectados.

O efeito da conexidade dos dados sobre a variância genética aditiva foi pequeno. Apenas os dados com $0 \%$ de conexidade apresentaram redução da variância genética aditiva, que diferiu dos demais graus de conexidade.

\section{Referências Bibliográficas}

ANALLA, M., SANCHEZ PALMA, A., MUÑOZ SERRANO, A., et al. 1995. Simulation analysis with BLUP methodology of different data structures in goat selection schemes in Spain. Small Ruminant Research,17(1):51-55.

CARNEIRO, P.L.S. Efeito de erros de pedigree na avaliação genética animal. Viçosa, MG: UFV, 1998. 79p. Dissertação (Mestrado em Genética e Melhoramento) - Universidade Federal de Viçosa, 1998.

EUCLYDES, R.F. Uso do sistema para simulação Genesys na avaliação de métodos de seleção clássicos e associados a marcadores moleculares. Viçosa, MG: UFV, 1996. 149p. Dissertação (Doutorado em Genética e Melhoramento) Universidade Federal de Viçosa, 1996.
FOULlEY, J.L., HANOCQ, E., BOICHARD, D. 1992. A criterion for measuring the degree of connectedness in linear models of genetic evaluation. Genetics-Selection-Evolution, 24(4):315-330.

KENNEDY, B.W. 1981. Bias and mean square error fron ignoring genetic groups in mixed model sire evaluation. J. Dairy Sci., 64(4):689-697.

KENNEDY, B.W., TRUS, D. 1993. Considerations on genetic connectedness between management units under an animal model. J. Anim. Sci., 71(9):2341-2352.

MARTINS, E.N. Uso de modelos mistos no melhoramento animal. In: SIMPÓSIO INTERNACIONAL DE PRODUÇÃO DE NÃO-RUMINANTES, 1994, Maringá. Anais... Maringá: SBZ, 1994. p.41-46.

MATHUR, P.K., SULLIVAN, B., CHESNAIS, J. "Estimation of the degree connectedness between herds or management groups in the canadian swine population", <http:// www.ccsi.ca/pramod/connectedness.htm> (07/01/99).

SEARLE, S.R. 1971. Linear models. New York: John Wiley \& Sons. 532p.

SIGURDSSON, A., BANOS, G. 1995. Dependent variables in international sire evaluations. Acta Agric. Scand., Section A, Animal Science, 45(4):209-217.

TONG, A.K.W., KENNEDY, B.W., MOXLEY, J.E. 1980. Potential errors in sire evaluation from regional genetic differences. J. Dairy Sci., 63(4):627-633.

Recebido em: 10/02/00

Aceito em: 23/11/00 